\title{
Obesity Indices Related to Obstructive Sleep Apnea in Obese Adults
}

\author{
Jongwoo Kim, Seon Yeong Lee* \\ Department of Family Medicine, Inje University Sanggye-Paik Hospital, Seoul, South Korea \\ Email: *sylee@paik.ac.kr
}

How to cite this paper: Kim, J. and Lee, S.Y. (2017) Obesity Indices Related to Obstructive Sleep Apnea in Obese Adults. Journal of Biosciences and Medicines, 5, 34-43.

https://doi.org/10.4236/jbm.2017.510004

Received: September 29, 2017

Accepted: October 21, 2017

Published: October 24, 2017

Copyright $\odot 2017$ by authors and Scientific Research Publishing Inc. This work is licensed under the Creative Commons Attribution International License (CC BY 4.0).

http://creativecommons.org/licenses/by/4.0/

\section{(c) (i) Open Access}

\begin{abstract}
Background: Obstructive Sleep Apnea (OSA) is prevalent in obese patients. OSA should be evaluated because it might increase mortality. We aimed to evaluate the risk for OSA in obese Korean adults, and to examine the obesity indices most strongly associated with OSA. Method: Anthropometric measurements, fat computed tomography (CT) and dual energy X-ray absorptiometry (DEXA) were performed in 30 obese patients. All patients were divided into low or high risk group of OSA using Berlin Questionnaire. We compared differences between the groups in obesity-related indices. Result: Eleven of the 30 patients (36.7\%) were in the low OSA risk group and 19 (63.3\%) were in the high OSA risk group. The correlation coefficients for BMI, neck circumference, and neck-to-height ratio $\times 100(\%)$ in the high-risk group versus the low-risk group were $1.03,1.96$, and 4.04 , respectively $(\mathrm{P}=$ 0.03). Conclusion: The obesity index most strongly associated with OSA was neck circumference to height ratio.
\end{abstract}

\section{Keywords}

Obesity, Sleep Apnea, Obstructive, Obesity Index, Neck Circumference to Height Ratio

\section{Introduction}

Obesity causes chronic disease including cardiovascular disease, diabetes and cancer, and $40 \%-90 \%$ of obese patients also exhibit signs and symptoms of sleep apnea [1]. Obesity-related obstructive sleep apnea (OSA) refers to repeated pharyngeal obstruction during sleep, which not only leads to negative consequences, such as fatigue, reduced cognitive ability, headache, depression and sexual dysfunction, but also increases the risk for hypertension, cardiovascular disease, arrhythmia, and heart failure. OSA, therefore, is one of the most important 
diseases to be assessed early in obese patients [2]. Risk factors associated with OSA include obesity, male sex, older age, neck circumference, facial dysmorphism, genetic factors, and thyroid disease [2].

Body mass index (BMI) is widely used as a measure of obesity; however, because it does not reflect the extent of obesity, it is difficult to use for diagnosing and evaluating the severity of OSA. Obesity and regional fat distribution, measured by neck fat mass percentage using dual-energy X-ray absorptiometry (DEXA), might correlate with obstructive sleep apnea (OSA) severity in adults [3], but Martin et al. reported the lack of correlation between DEXA measurements changes and the changes in the apnea hypopnea index [4]. There are still conflicting opinions among researchers regarding the factors that enable easy assessment of OSA, and a lack of research in this regard in Korea remains. Accordingly, the present study aimed to evaluate the risk for OSA in obese Korean adults, and to examine the obesity indices most strongly associated with OSA.

\section{Methods}

\subsection{Subjects}

Obese adults who visited the obesity clinic at a single university hospital for an initial consultation between March and July 2013 were investigated. Thirty-four patients underwent an initial consultation during the study period; however, DEXA could not be performed in 4 individuals due to super-morbid obesity; therefore, 30 patients were included in the final analysis.

\subsection{Anthropometric Measurements, DEXA, and Fat Computed Tomography}

Patient height, weight, neck circumference, and waist circumference were measured at the first visit. Neck circumference was adjusted by separately calculating the neck-to-height ratio (neck circumference divided by height [both in $\mathrm{cm}$ )). Anthropometric measurements were each performed twice by a trained specialist, and the average of the two measurements was used. Height was measured in $\mathrm{cm}$ and weight was measured in $\mathrm{kg}$. Neck circumference was measured in $\mathrm{cm}$, in the horizontal plane passing through the center of the cricothyroid membrane; waist circumference was measured in $\mathrm{cm}$ at the widest point below the navel.

Fat computed tomography (CT) and DEXA were performed in all patients. For fat CT, an Aquilion ONE (Toshiba, Otawara, Japan) device scanned a transverse section at the level of the navel (transverse cut), the Terarecon iNtuition program was used to select fat as areas of -190 to -30 Hounsfield units, and the areas of visceral and subcutaneous fat were calculated. Total body fat mass and regional fat mass were measured using a fan-beam densitometer (Lunar Prodigy, GE Medical Systems, Diegem, Belgium). Body fat corresponded to body weight in $\mathrm{kg}$ minus lean mass and bone mineral content. Arm, leg, trunk, android, and gynoid fat mass were calculated based on specific anatomical position, according to guidelines from the manufacturers of the DEXA instrument. To determine 
the regional distribution of total body fat mass, the regional fat mass values measured using DEXA were divided by the total body fat mass and divided by 100 to obtain a percentage $(\%)$.

\subsection{Berlin Questionnaire}

The Berlin questionnaire was used to evaluate OSA risk [5]. This questionnaire consists of 10 questions divided into three categories. The first category consists of 5 questions on snoring and sleep apnea; answering positively to at least 2 questions is considered to be a positive result for the category. The second category consists of 4 questions on weekly tiredness; answering positively to at least 2 questions is considered to be a positive result for the category. The third category queries whether the subject has hypertension or a BMI of at least $30 \mathrm{~kg} / \mathrm{m}^{2}$. Subjects with positive results in at least 2 of the 3 categories are classified as high risk for OSA, and other subjects are classified as low risk [1] [5]. Because most of the subjects in the present study were severely obese, the BMI criterion was arbitrarily set at $30 \mathrm{~kg} / \mathrm{m}^{2}$.

\subsection{Statistical Analysis}

Patients in this study were divided into two groups according to OSA risk, and the Mann-Whitney test was used to compare differences between the groups in obesity-related indices. Correcting for age and sex, a logistic regression analysis was performed to examine the effects of obesity-related indices on OSA risk, and the regression coefficients and significance probabilities were calculated for each variable. SAS version 8.1 (SAS Institute, Cary, NC, USA) was used for statistical analysis and $\mathrm{P}<0.05$ was considered to be statistically significant.

\section{Results}

\subsection{Comparison between Males and Females}

The 30 adult obese patients were divided according to sex, and the mean and standard deviation were calculated for each variable (Table 1). For males and females, respectively, the mean ages were $32.4 \pm 13.0$ years and $36.8 \pm 10.5$ years; the mean BMI was $33.3 \pm 4.4 \mathrm{~kg} / \mathrm{m}^{2}$ and $30.5 \pm 3.8 \mathrm{~kg} / \mathrm{m}^{2}$. The mean neck circumferences for males and females, respectively, were $43.2 \pm 2.7 \mathrm{~cm}$ and $36.1 \pm$ $2.8 \mathrm{~cm}$, the mean neck-to-height ratios were $0.25 \pm 0.02$ and $0.23 \pm 0.01$, and the mean waist circumferences were $109.9 \pm 12.8 \mathrm{~cm}$ and $100.6 \pm 8.8 \mathrm{~cm}$. Abdominal visceral fat area, as measured by fat CT, was $197.1 \pm 72.7 \mathrm{~cm}^{2}$ for males and $143.4 \pm 64.3 \mathrm{~cm}^{2}$ for females. Body fat percentage measured using DEXA was $33.1 \% \pm 5.1 \%$ in males and $41.3 \% \pm 5.2 \%$ in females, the leg fat distribution was $28.9 \% \pm 4.2 \%$ and $32.2 \% \pm 5.7 \%$ in males and females, respectively, and the trunk fat distribution was $59.7 \% \pm 3.5 \%$ and $56.0 \% \pm 5.7 \%$.

\subsection{Comparison between High and Low OSA Risk Groups}

Based on the Berlin questionnaire, 11 of the 30 patients $(36.7 \%)$ were in the low 
Table 1. Basic characteristics of subjects.

\begin{tabular}{|c|c|c|}
\hline & Male $(\mathrm{n}=10)$ & Female $(\mathrm{n}=20)$ \\
\hline Age, y & $32.4 \pm 13.0$ & $36.8 \pm 10.5$ \\
\hline Height, $\mathrm{cm}$ & $174.1 \pm 8.4$ & $158.8 \pm 6.2$ \\
\hline Body weight, $\mathrm{kg}$ & $101.0 \pm 18.3$ & $77.1 \pm 13.0$ \\
\hline $\mathrm{BMI}, \mathrm{kg} / \mathrm{m}^{2}$ & $33.3 \pm 4.4$ & $30.5 \pm 3.8$ \\
\hline Neck circumference, $\mathrm{cm}$ & $43.2 \pm 2.7$ & $36.1 \pm 2.8$ \\
\hline Neck to height ratio ${ }^{\dagger}$ & $0.25 \pm 0.02$ & $0.23 \pm 0.01$ \\
\hline Waist circumference, $\mathrm{cm}$ & $109.9 \pm 12.8$ & $100.6 \pm 8.8$ \\
\hline Abdomen viasceral fat area ${ }^{\ddagger}, \mathrm{cm}^{2}$ & $197.1 \pm 72.7$ & $143.4 \pm 64.3$ \\
\hline Abdomen subcutaneous fat area ${ }^{\ddagger}, \mathrm{cm}^{2}$ & $336.1 \pm 145.9$ & $311.0 \pm 67.4$ \\
\hline Visceral to subcutaneous fat ratio & $0.7 \pm 0.3$ & $0.5 \pm 0.2$ \\
\hline \multicolumn{3}{|l|}{ Fat mass ${ }^{\mathrm{II}}, \mathrm{kg}$} \\
\hline Arm & $2.8 \pm 0.8$ & $2.8 \pm 0.8$ \\
\hline Leg & $10.1 \pm 4.2$ & $10.4 \pm 3.0$ \\
\hline Trunk & $20.2 \pm 6.2$ & $17.8 \pm 4.1$ \\
\hline Android & $3.8 \pm 1.4$ & $3.0 \pm 0.9$ \\
\hline Gynoid & $5.6 \pm 2.0$ & $5.6 \pm 1.3$ \\
\hline Total & $34.0 \pm 11.0$ & $32.0 \pm 7.1$ \\
\hline \multicolumn{3}{|l|}{ Fat distribution ${ }^{\mathrm{II}, \boldsymbol{9}}, \%$} \\
\hline Arm & $8.4 \pm 0.9$ & $8.5 \pm 1.1$ \\
\hline Leg & $28.9 \pm 4.2$ & $32.2 \pm 5.7$ \\
\hline Trunk & $59.7 \pm 3.5$ & $56.0 \pm 5.7$ \\
\hline Android & $11.1 \pm 1.2$ & $9.3 \pm 1.5$ \\
\hline Gynoid & $16.3 \pm 1.3$ & $17.4 \pm 2.0$ \\
\hline Total & $33.1 \pm 5.1$ & $41.3 \pm 5.2$ \\
\hline
\end{tabular}

Abbreviations: BMI, body mass index calculated as weight in kilograms divided by height in meters squared. ${ }^{\dagger}$ Neck to height ratio calculated as neck circumference in centimeters divided by height in centimeters. ${ }^{*}$ Measurements by fat computed tomography. ${ }^{5}$ Visceral to subcutaneous fat ratio calculated with abdomen visceral fat area in centimeters squared divided by abdomen subcutaneous fat area in centimeters squared. IIMeasurements bydual energy X-ray absorptiometry scan. 'Fat distribution calculated as each regional fat mass divided by total fat mass and multiplied by 100 .

OSA risk group and 19 (63.3\%) were in the high OSA risk group (Table 2). The mean age was $31 \pm 9.0$ years in the low OSA risk group and $37.8 \pm 12.0$ years in the high OSA risk group, and the proportion of males was $18 \%$ and $42 \%$, respectively. BMI was $29.3 \pm 3.8 \mathrm{~kg} / \mathrm{m}^{2}$ in the low risk group and $32.6 \pm 4.0 \mathrm{~kg} / \mathrm{m}^{2}$ in the high risk group, neck-to-height ratio was $0.22 \pm 0.01$ and $0.24 \pm 0.02$, respectively, and waist circumference was $99.3 \pm 11.3 \mathrm{~cm}$ and $106.2 \pm 10.3 \mathrm{~cm}$. Abdominal visceral fat area was $125.3 \pm 39.1 \mathrm{~cm}^{2}$ in the low OSA risk group and $182.1 \pm 77.3 \mathrm{~cm}^{2}$ in the high OSA risk group, while the abdominal visceral-to-subcutaneous fat ratio was $0.4 \pm 0.2$ and $0.6 \pm 0.3$, respectively. The leg fat distribution was $33.8 \% \pm 5.5 \%$ in the low risk group and $29.5 \% \pm 4.8 \%$ in the high risk group, the trunk fat distribution was $54.8 \% \pm 5.3 \%$ and $58.6 \% \pm 4.9 \%$ 
Table 2. The comparison of variables according to OSA risk.

\begin{tabular}{|c|c|c|c|}
\hline & $\begin{array}{l}\text { OSA low risk } \\
\qquad(\mathrm{n}=11)\end{array}$ & $\begin{array}{l}\text { OSA high risk } \\
\qquad(\mathrm{n}=19)\end{array}$ & $\mathrm{P}^{*}$ \\
\hline Number (male, female) & 2,9 & 8,11 & \\
\hline Age, y & $31.0 \pm 9.0$ & $37.8 \pm 12.0$ & 0.13 \\
\hline Height, $\mathrm{cm}$ & $161.8 \pm 9.4$ & $165.0 \pm 10.5$ & 0.35 \\
\hline Body weight, kg & $78.0 \pm 20.2$ & $89.1 \pm 16.9$ & 0.04 \\
\hline $\mathrm{BMI}, \mathrm{kg} / \mathrm{m}^{2}$ & $29.3 \pm 3.8$ & $32.6 \pm 4.0$ & 0.02 \\
\hline Neck circumference, $\mathrm{cm}$ & $35.9 \pm 3.6$ & $40.0 \pm 4.1$ & 0.01 \\
\hline Neck to height ratio ${ }^{\dagger}$ & $0.22 \pm 0.01$ & $0.24 \pm 0.02$ & $<0.01$ \\
\hline Waist circumference, $\mathrm{cm}$ & $99.3 \pm 11.3$ & $106.2 \pm 10.3$ & 0.04 \\
\hline Abdomen viasceral fat area ${ }^{\ddagger}, \mathrm{cm}^{2}$ & $125.3 \pm 39.1$ & $182.1 \pm 77.3$ & 0.03 \\
\hline Abdomen subcutaneous fat area ${ }^{\ddagger}, \mathrm{cm}^{2}$ & $316.7 \pm 114.8$ & $320.8 \pm 91.4$ & 0.43 \\
\hline Visceral to subcutaneous fat ratio ${ }^{\ddagger, \S}$ & $0.4 \pm 0.2$ & $0.6 \pm 0.3$ & 0.03 \\
\hline \multicolumn{4}{|l|}{ Fat mass ${ }^{\mathrm{II}}, \mathrm{kg}$} \\
\hline Arm & $2.5 \pm 0.6$ & $2.9 \pm 0.8$ & 0.16 \\
\hline Leg & $10.7 \pm 3.3$ & $10.1 \pm 3.4$ & 0.85 \\
\hline Trunk & $17.4 \pm 5.1$ & $19.3 \pm 4.8$ & 0.07 \\
\hline Android & $2.9 \pm 1.3$ & $3.4 \pm 1.1$ & 0.06 \\
\hline Gynoid & $5.7 \pm 1.5$ & $5.6 \pm 1.6$ & 0.85 \\
\hline Total & $31.6 \pm 8.4$ & $33.3 \pm 8.7$ & 0.25 \\
\hline \multicolumn{4}{|l|}{ Fat distribution ${ }^{\mathrm{II}, \mathscr{9}}, \%$} \\
\hline Arm & $8.1 \pm 1.1$ & $8.7 \pm 0.9$ & 0.11 \\
\hline Leg & $33.8 \pm 5.5$ & $29.5 \pm 4.8$ & 0.03 \\
\hline Trunk & $54.8 \pm 5.3$ & $58.6 \pm 4.9$ & 0.04 \\
\hline Android & $9.1 \pm 1.6$ & $10.3 \pm 1.5$ & 0.04 \\
\hline Gynoid & $17.9 \pm 2.0$ & $16.5 \pm 1.6$ & 0.06 \\
\hline Total & $40.7 \pm 3.9$ & $37.4 \pm 7.4$ & 0.29 \\
\hline
\end{tabular}

${ }^{*} \mathrm{P}$ value from Mann-Whitney test. Subjects with positive results in at least 2 of the 3 categories of Berlin questionnaire were classified as high risk for OSA, and other subjects were classified as low risk. Abbreviations: OSA, Obstructive sleep apnea. BMI, body mass index calculated as weight in kilograms divided by height in meters squared. ${ }^{\dagger}$ Neck to height ratio calculated as neck circumference in centimeters divided by height in centimeters. ${ }^{\neq}$Measurements by fat computed tomography. ${ }^{5}$ Visceral to subcutaneous fat ratio calculated as abdomen visceral fat area in centimeters squared divided by abdomen subcutaneous fat area in centimeters squared. IIMeasurements by dual energy X-ray absorptiometry scan. 'Fat distribution was calculated with each regional fat mass divided by total fat mass and multiplied by 100 .

respectively, and the android fat distribution was $9.1 \% \pm 1.6 \%$ and $10.3 \% \pm 1.5 \%$.

\subsection{Regression Analysis}

The results of regression analysis of obesity-related indices on OSA risk are summarized in Table 3. The correlation coefficients for BMI, neck circumference, and neck-to-height ratio $\times 100(\%)$ in the high-risk group versus the low-risk group were $1.03,1.96$, and 4.04 , respectively. 
Table 3. Logistic regression analysis of obesity indices on OSA risk.

\begin{tabular}{|c|c|c|c|}
\hline & $\begin{array}{l}\text { Regression } \\
\text { coefficient }\end{array}$ & $\mathrm{P}^{*}$ & $95 \%$ CI \\
\hline Body weight, kg & 1.07 & 0.12 & $0.98-1.16$ \\
\hline $\mathrm{BMI}, \mathrm{kg} / \mathrm{m}^{2}$ & 1.03 & 0.03 & $1.03-2.03$ \\
\hline Neck circumference, $\mathrm{cm}$ & 1.96 & 0.04 & $1.02-3.75$ \\
\hline Neck to height ratio ${ }^{\dagger}, \%$ & 4.04 & 0.03 & $1.20-13.65$ \\
\hline Waist circumference, $\mathrm{cm}$ & 1.09 & 0.14 & $0.97-1.21$ \\
\hline Abdomen visceral fat $\operatorname{area}^{\ddagger}, \mathrm{cm}^{2}$ & 1.02 & 0.16 & $0.99-1.04$ \\
\hline Abdomen visceral to subcutaneous fat ratio & 8.93 & 0.35 & $0.91-874.21$ \\
\hline \multicolumn{4}{|l|}{ Fat distribution ${ }^{\mathrm{II}, 9}, \%$} \\
\hline Leg & 0.91 & 0.38 & $0.74-1.12$ \\
\hline Trunk & 1.06 & 0.61 & $0.86-1.30$ \\
\hline Android & 1.31 & 0.43 & $0.67-2.57$ \\
\hline
\end{tabular}

${ }^{\star} \mathrm{P}$ value from logistic regression analysis adjusted by age and sex. Abbreviations: OSA, Obstructive sleep apnea. BMI, body mass index calculated as weight in kilograms divided by height in meters squared. CI, Confidence interval. ${ }^{\dagger} \mathrm{Neck}$ to height ratio (\%) calculated as neck circumference divided by height and multiplied by $100 .{ }^{*}$ Measurements by fat computed tomography. ${ }^{5}$ Visceral to subcutaneous fat ratio calculated as abdomen visceral fat area in centimeters squared divided by abdomen subcutaneous fat area in centimeters squared. IIMeasurements by dual energy X-ray absorptiometry scan. 'Fat distribution was calculated with each regional fat mass divided by total fat mass and multiplied by 100 .

\section{Discussion}

In the present study, we used the Berlin questionnaire to examine 30 adult patients with obesity at a single university hospital, and found that 19 (63\%) were in the high OSA risk group. Compared with the low OSA risk group, the high-risk group exhibited a greater proportion of male patients and older age; however, these differences were not statistically significant. BMI, neck circumference, neck-to-height ratio, abdominal visceral fat area, abdominal visceral-tosubcutaneous fat ratio, trunk fat distribution, and android fat distribution were all significantly higher in the high OSA risk group.

Accurate diagnosis of OSA requires subjects to undergo polysomnography and be assigned a score of at least 5 on the apnea hypopnea index (AHI); however, the costs, in terms of time and money, make this impractical in primary care settings. For this reason, various studies have explored methods to replace polysomnography. In the present study, we used the Berlin questionnaire to classify OSA risk. This questionnaire was developed at the Conference on Sleep in Primary Care, held in Berlin (Germany) in 1996 and, to date, is the most widely used questionnaire for OSA screening. There have been numerous studies investigating the predictive accuracy of the Berlin questionnaire for OSA, and its utility has been demonstrated in a study involving the general population in Korea [6]. However, the results were not significant in a study involving patients at a sleep clinic [7], and studies involving obese patients have yet to be conducted. 
In studies investigating OSA, BMI and neck circumference have been reported to be the strongest predictive factors for apnea-hypopnea [8] [9] [10] [11]. Meanwhile, other studies have reported that body fat and abdominal circumference are the factors that best reflect OSA risk [12] [13]. In one investigation, the index most closely related to OSA risk was abdominal visceral fat in middle-age males and neck circumference in females [14].

Unlike previous studies, our study revealed no significant difference between the OSA risk groups in sex ratio or age; we believe this reflected the characteristics of the subject population. In one study [8], the mean age of the patients was the sixth decade of life (i.e., $50 \mathrm{~s}$ ), and the majority were male. In our study, the mean age of the patients was the fourth decade of life (i.e., $30 \mathrm{~s}$ ), and there were twice as many females as males. However, the previous study [8] involved patients who visited a sleep clinic for snoring, whereas our study focused on patients visiting an obesity clinic for weight loss. Because most of the patients at the sleep clinic were not obese, their complaints were more likely to be the result of anatomical issues in the head and neck, such as tonsillar hypertrophy or a small mandible, which may explain the difference in results.

Considering the nature of the obese patient group in the present study, we performed a regression analysis corrected for sex and age, which have been shown to affect OSA in previous studies. The variable that exhibited the largest significant correlation coefficient in the high OSA risk group was neck-to-height ratio $\times 100(\%)$. The reason for converting neck-to-height ratio into a percentage (by multiplying by 100) is because, without this change, the correlation coefficient was too large, in the order of 1060 . Because the regression coefficient for the neck-to-height ratio (\%) was 4 , this means that an increase in the neck circumference equal to $1 \%$ of the height will increase the likelihood of inclusion in the high OSA risk group by four-fold. One possible reason for patients with larger necks having an increased risk for OSA is the relationship with local fat accumulation. Because our study involved patients with a BMI of $\geq 25 \mathrm{~kg} / \mathrm{m}^{2}$, accumulation of fat in the tongue and laryngopharynx could constrict the diameter of the trachea, potentially leading to obstruction during sleep [14]. We did not observe significant results for abdominal visceral fat area or regional fat distribution.

We attempted a stepwise regression analysis using variables other than age and sex; however, the results were not statistically significant. Consequently, it is difficult to determine which variables are the strongest predictors. Because other variables that are potential covariates are, in large part, obesity-related indices, there is some overlap, which can cause problems in the regression formula. For example, an individual with high body weight will likely have a broader abdominal fat area, while in DEXA results, trunk fat includes android fat. These issues will need to be analyzed in a study involving a larger sample size.

One of the strengths of our study was that all the subjects were obese, with a mean BMI $>30 \mathrm{~kg} / \mathrm{m}^{2}$. Because previous studies have mostly focused on sleep clinic patients, the results cannot be directly applied to obese patients. Based on 
the results of our study, we confirmed that $60 \%-70 \%$ of obese Korean patients are at high risk for OSA. Moreover, given that there is a close association between high OSA risk and neck-to-height ratio, and that conducting polysomnography in all obese patients is impractical, measuring neck circumference could be helpful in predicting OSA risk. Second, previous research reporting that abdominal obesity is the strongest predictive factor for OSA [5] used abdominal circumference and bioelectrical impedance analysis. However, in our study, we were able to analyze fat distribution more precisely using DEXA, which is considered to be the standard method for body fat analysis, and fat CT, which can separately determine areas of abdominal visceral and subcutaneous fat. Third, in previous studies that used DEXA measurements to investigate fat distribution [15] [16], the distribution was calculated as regional fat weight/regional weight $\times$ $100(\%)$, or as regional fat weight/total body weight $\times 100(\%)$. In contrast, our study used regional fat weight/total body fat weight $\times 100(\%)$. Calculating the regional fat distribution as a percentage of total body fat has the effect of normalizing according to total body fat, enabling meaningful comparisons of fat distribution according to region, even among patients with the same total body fat. This differentiates our study from others and improves the validity of our results.

Our study also had some limitations, the first of which were the small sample size and the low proportion of male subjects. Second, because this was a cross-sectional study, we were unable to clearly demonstrate causality. Third, we were unable to use polysomnography to accurately diagnose OSA; therefore, we used the Berlin questionnaire instead. Although the Berlin questionnaire is the best method to replace polysomnography for OSA screening, the effectiveness of the questionnaire for testing obese Korean patients has not yet been demonstrated, which limits the interpretation and applicability of our results. In addition, because the Berlin questionnaire evaluates OSA risk as a categorical variable (high risk versus low risk), we were only able to perform a logistic regression analysis. If polysomnography had been used to obtain AHI values, it would be possible to derive more precise results using linear regression analysis. Because of these limitations, further research will need to be conducted in the future, studying more patients and over a longer duration. To investigate whether larger neck circumference in the high OSA risk group was actually due to fat distribution in the neck, neck fat would need to be measured and analyzed using DEXA, similar to the study by Simpson et al. [14]. In addition, follow-up observation of the patients in this study to investigate changes in obesity-related factors with weight loss would help to verify whether this also reduces OSA risk.

The effectiveness of the Berlin questionnaire needs to be demonstrated in obese Korean patients. The second category of the survey was shown to have a weaker association with OSA in a study by Kang et al. [7]. If this category was excluded and the other questions were combined to calculate scores, we believe the Berlin questionnaire could be used widely for convenient screening of patients-without polysomnography-to determine the course of future examina- 
tions, and to evaluate the effects of intervention(s).

\section{Conflict of Interest}

We declare that we have no conflict of interest.

\section{Ethical Approval}

This study was approved by the Institutional Review Board of Inje University Sanggye Paik Hospital in Seoul, South Korea.

\section{References}

[1] Alan, R.S., Susheel, P.P., Alison, M.L., Vsevolod, P., Hartmut, S. and Philip, L.S. (2008) Obesity and Obstructive Sleep Apnea. Proceedings of the American Thoracic Society, 5, 185-192. https://doi.org/10.1513/pats.200708-137MG

[2] Neomi, S. and Francoise, R. (2009) The Relationship of Obesity and Obstructive Sleep Apnea. Clinics in Chest Medicine, 20, 455-465.

[3] Glicksman, A., Hadjiyannakis, S., Barrowman, N., Walker, S., Hoey, L. and Katz, S.L. (2017) Body Fat Distribution Ratios and Obstructive Sleep Apnea Severity in Youth With Obesity. Journal of Clinical Sleep Medicine, 13, 545-550. https://doi.org/10.5664/jcsm.6538

[4] Martin, S.M., Roche, F., Thomas, T., Collet, P., Barthélémy, J.C. and Sforza, E. (2015) Association of Body Fat Composition and Obstructive Sleep Apnea in the Elderly: A Longitudinal Study. Obesity, 23, 1511-1516. https://doi.org/10.1002/oby.21121

[5] Netzer, N.C., Stoohs, R.A., Netzer, C.M., Clark, K. and Strohl, K.P. (1999) Using the Berlin Questionnaire to Identify Patients at Risk for the Sleep Apnea Syndrome. Annals of Internal Medicine, 131, 485-491. https://doi.org/10.7326/0003-4819-131-7-199910050-00002

[6] Kang, K., Park, K.S., Kim, J.E., Kim, S.W., Kim, Y.T., Kim, J.S. and Lee, H.W. (2013) Usefulness of the Berlin Questionnaire to Identify Patients at High Risk for Obstructive Sleep Apnea: A Population-Based Door-to-Door Study. Sleep and Breathing, 17, 803-810. https://doi.org/10.1007/s11325-012-0767-2

[7] Kang, H.H., Kang, J.Y., Lee, S.H. and Moon, H.S. (2011) The Usefulness of the Berlin Questionnaire as a Screening for Obstructive Sleep Apnea in a Sleep Clinic Population. Sleep Medicine and Psychophysiology, 18, 82-86.

[8] Han, S.J., Joo, E.Y., Kim, J.H., Kim, M.S. and Hong, S.B. (2004) Body Mass Index and Neck Circumference in Patients with Obstructive Sleep Apena-Hypopnea Syndromes. Journal of Korean Sleep Research, 1, 37-41. https://doi.org/10.13078/jksrs.04016

[9] Yeh, P.S., Lee, Y.C., Lee, W.J., Chen, S.B., Ho, S.J., Peng, W.B., et al. (2010) Clinical Predictors of Obstructive Sleep Apnea in Asian Bariatric Patients. Obesity Surgery, 20, 30-35. https://doi.org/10.1007/s11695-009-9854-2

[10] Hari, L.P., Rajagopal, T., James, P. and Ravindran, C. (2012) Clinical Prediction of OSA in a Tertiary Care Setting. JCDR, 6, 835-838.

[11] Soriano-Co, M., Vanhecke, T.E., Franklin, B.A., Sangal, R.B., Hakmeh, B. and McCullough, P.A. (2011) Increased Central Adiposity in Morbidly Obese Patients with Obstructive Sleep Apnoea. Internal Medicine Journal, 41, 560-566. https://doi.org/10.1111/j.1445-5994.2010.02283.x 
[12] Lovin, S., Bercea, R., Cojocaru, C., Rusu, G. and Mihăescu, T. (2010) Body Composition in Obstructive Sleep Apnea Hypopnea Syndrome Bio-Impedance Reflects the Severity of Sleep Apnea. Multidisciplinary Respiratory Medicine, 5, 44-49. https://doi.org/10.1186/2049-6958-5-1-44

[13] Shinohara, E., Kihara, S., Yamashita, S., Yamane, M., Nishida, M., Arai, T., et al. (1997) Visceral Fat Accumulation as an Important Risk Factor for Obstructive Sleep Apnoea Syndrome in Obese Subjects. Journal of Internal Medicine, 241, 11-18. https://doi.org/10.1046/j.1365-2796.1997.63889000.x

[14] Li, Y., Lin, N., Ye, J., Chang, Q., Han, D. and Sperry, A. (2012) Upper Airway Fat Tissue Distribution in Subjects with Obstructive Sleep Apnea and Its Effect on Retropalatal Mechanical Loads. Respiratory Care, 57, 1098-1105.

https://doi.org/10.4187/respcare.00929

[15] Simpson, L., Mukherjee, S., Cooper, M.N., Ward, K.L., Lee, J.D., Fedson, A.C., et al. (2010) Sex Differences in the Association of Regional Fat Distribution with the Severity of Obstructive Sleep Apnea. Sleep, 33, 467-474.

https://doi.org/10.1093/sleep/33.4.467

[16] Bezerra, P.C., do Prado, M., Gaio, E., Franco, O.L. and Tavares, P. (2013) The Use of Dual-Energy X-Ray Absorptiometry in the Evaluation of Obesity in Women with Obstructive Sleep Apnea-Hypopnea Syndrome. European Archives of Oto-RhinoLaryngology, 270, 1539-1545. https://doi.org/10.1007/s00405-012-2291-1 